\title{
A Classification of Sitting Strategies based on Driving Posture Analysis
}

\author{
Jangwoon Park ${ }^{1}$, Younggeun Choi ${ }^{1}$, Baekhee Lee ${ }^{1}$, Kihyo Jung ${ }^{2}$, Sungjin Sah ${ }^{3}$, Heecheon You ${ }^{1}$ \\ ${ }^{1}$ POSTECH; Department of Industrial and Management Engineering, Gyeongbuk, 790-784 \\ ${ }^{2}$ University of Ulsan; School of Industrial Engineering \\ ${ }^{3}$ HYUNDAI MOTOR; Research \& Development Division
}

\section{Corresponding Author}

Heecheon You

POSTECH, Department of Industrial and

Management Engineering, 77 Cheongam-

Ro, Nam-Gu, Pohang, Gyeongbuk, 790-

784

Mobile : +82-10-3213-2210

Email : hcyou@postech.ac.kr

Received : January 09, 2014

Revised : February 06, 2014

Accepted: April 03, 2014
Objective: The present study is intended to objectively classify upper- \& lower-body sitting strategies and identify the effects of gender and OPL type on the sitting strategies.

Background: A sitting strategy which statistically represents comfortable driving posture can be used as a reference posture of a humanoid in virtual design and evaluation of a driver's seat. Although previous research has classified sitting strategies for driving postures in various occupant package layout (OPL) types, the existing classification methods are not objective and the factors affecting sitting strategies have not been identified.

Method: Forty drivers' preferred driving postures in three different OPL types (coupe, sedan, and SUV) were measured by a motion capture system. Next, the measured driving postures were classified by K-means cluster method.

Results: Sitting strategies of upper-body were classified as erect (33\%), slouched (41\%), and reclined (26\%) postures, and those of lower-body were classified as knee bent (42\%), knee extended (32\%), and upper-leg lifted (26\%) postures. Significant differences at $\alpha=0.05$ in the upper-body sitting strategy by gender and lowerbody sitting strategy by OPL type were found.

Application: Both the classified sitting strategies and the identified factors would be of use in ergonomic seat design and evaluation.

Keywords: Sitting strategy, Driving posture, Occupant package layout, Motion capture system

\section{Introduction}

Various research about driving posture have been conducted to design an ergonomics driver seat. Driving posture is a major factor for driving performance and seating comfort; as important information to design a driver seat. For example, driving posture is used as a reference data to design visibility, reachability, and affordable clearance of a driver workspace (Andreoni et al., 2002), and a driver seat for reducing back muscle fatigue (Grieco, 1986). Park et al. (2000) analyzed the relationship between driving posture and seat configuration, Kyung and Nussbaum (2009) analyzed the preferred range of joint angles (neck, shoulder, elbow, wrist, torso, hip, knee, and ankle angles). Research interests in driver seat design/evaluation of a virtual

\footnotetext{
Copyright@2014 by Ergonomics Society of Korea. All right reserved.

Q This is an open-access article distributed under the terms of the Creative Commons Attribution Non-Commercial License (http:// creativecommons.org/licenses/by-nc/3.0/), which permits unrestricted non-commercial use, distribution, and reproduction in any medium, provided the original work is properly cited.
} 
environment using digital human models (DHM) have been increasing: for example, Park et al. (2010) evaluated the predicted driving posture of RAMSIS ${ }^{\circledR}$, and Reed et al. (2002) developed statistical models to predict driving postures of DHMs.

Several studies have been conducted to identify preferred driving postures for ergonomic driver seat design/evaluation. Andreoni et al. (2002) defined "sitting strategy" as a class of sitting posture, and classified sitting strategies (upper body: lumbar, dorsal, and dorsal scapular strategy; lower body: ischiatic, intermediate, and trochanteric strategy) based on visually analyzed seating pressure characteristics of seatback and seatpan for 8 males. Choi et al. (2013) quantitatively classified sitting strategies for upper-body (mid-back and scapular, mid-back and lumbar, lumbar strategies) and lower-body (hip concentrated, hip and mid-thigh concentrated, hip and mid-thigh distributed strategies) using the body pressure ratios (BPR) for 40 adults. Park (2006) classified representative driving postures by cluster analysis on measured joint angles of 128 Korean male drivers in their preferred driving postures to use digital human model (DHM).

The identification method for sitting strategies in the previous study is not objective and factors for the sitting strategies were not clearly identified yet. Andreoni et al. (2002) visually classified the sitting strategies based on the seating pressure distribution analysis; however, since they analyzed the seating pressure distribution visually, the objectiveness of the classification method is insufficient. Although Park (2006) objectively identified the sitting strategies based on drivers' sitting postures, the subjects were only males and the factors (e.g., gender, OPL condition) for the sitting strategies were not analyzed.

The present study is intended to objectively classify sitting strategies based on drivers' preferred postures and identify factors for sitting strategies. The preferred driving postures of various body size drivers were measured in 3 occupant package layout (OPL) conditions (coupe, sedan, and SUV) using a motion capture system. The measured driving postures were statistically classified using a cluster analysis. The present study also analyzed the gender and OPL condition effect on the identified sitting strategies.

\section{Methods}

\subsection{Participants}

Forty drivers ( 20 males and 20 females) in their 20 s to 50 s with more than 2 years of driving experience were participated. The participants were recruited evenly for three stature groups (small: $<33^{\text {rd }} \%$ ile, medium: $33^{\text {rd }} \sim 66^{\text {th }} \%$ ile, large: $>66^{\text {th }} \%$ ile) formed by referring to corresponding Size Korea (2010) male and female anthropometric data ( 6 males for small, 6 for medium, and 8 for large group; 5 females for small, 8 females for medium, and 7 females for large group). The average stature of males was $173 \mathrm{~cm}(\mathrm{SD}=6.2$, range $=157 \sim 181 \mathrm{~cm})$ and that of the females was $161 \mathrm{~cm}(S D=5.8$, range $=150 \sim 170 \mathrm{~cm})$.

\subsection{Measurement of driving postures using motion capture system}

A reconfigurable seating buck was constructed and a motion capture system were used in the experiment (see Figure 1). The seating buck can be reconfigured for various OPL conditions (coupe, sedan, and SUV) by adjusting seat (e.g., seat height $=176 \mathrm{~mm}$ for coupe, $240 \mathrm{~mm}$ for sedan, and $305 \mathrm{~mm}$ for SUV), steering wheel, accelerator pedal, and brake locations. An Equus seat (Hyundai-Kia Motors, Korea) was installed into the seating buck, it has diverse adjustment capabilities such as seat position (forward/backward and upward/downward), cushion length, cushion angle, seatback angle, and headrest position. Lastly, six motion capture cameras (Hawk-I, Motion Analysis Co., USA) were used to capture driving postures (sampling rate $=60 \mathrm{~Hz}$ ).

The driving postures of the participants in the seating buck were measured in three steps (preparation, measurement, and debriefing). In the preparation step, the research purpose and experimental process were introduced to the participant who then signed a written informed consent form. Next, 21 anthropometric dimensions of the participant were obtained by following 


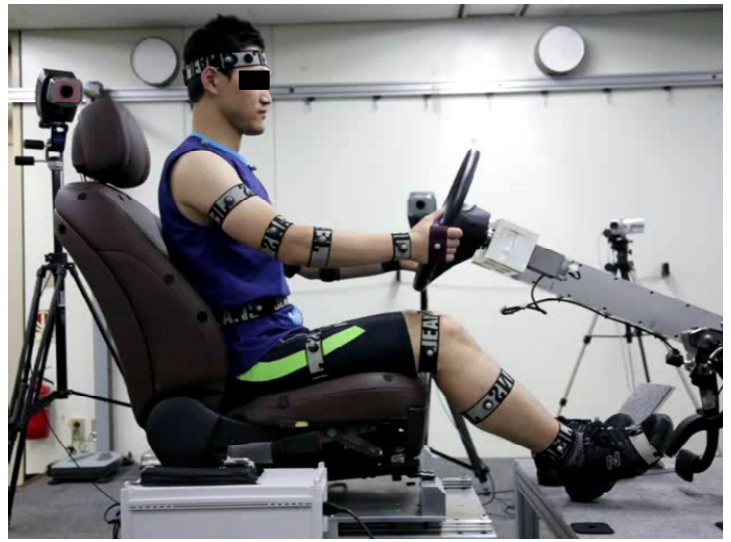

Figure 1. Measurement of driving postures using motion capture system

RAMSIS anthropometry protocol (Speyer, 2005) using a Martin's anthropometer (TTM, Tsutsumi Co., Japan). Repeated measurements were obtained for each anthropometric dimension until their differences become $<5 \mathrm{~mm}$. Next, 26 reflective markers $(\phi=$ $1.2 \mathrm{~cm}$ ) were attached on whole body of participant. In the measurement step, the participant was asked to sit on the driver mock-up with his/her preferred seating position and adjust seat configurations during 10-minute self-adjustment driving. After 10-minute self-adjustment driving, the preferred driving posture with both hands on 3-to-9 steering wheel positions was measured using the motion capture system in coupe, sedan, and SUV conditions. Lastly, a debriefing was conducted and the participant was compensated.

The measured driving postures of the participants were reconstructed in three steps (generation of reference points, adjustment of reference point locations, and synchronization of reference points and measured markers) using RAMSIS ${ }^{\circledR}$ (Human Solution $\mathrm{GmbH}$, Germany) humanoid. In the generation of reference point step, reference points on a humanoid were generated based on the attached marker locations on a participant's whole body. In the adjustment of reference point location step, the generated reference points on the humanoid whole body were relocated based on the distances between the each measured marker location. In the synchronization of reference point and measured marker step, the adjusted reference points on the humanoid were used to synchronize with the measured markers in driving posture using RAMSIS Animation Simulator (38 reference points can be synchronized with measured markers at once) in order to reconstruct each participant's driving posture using RAMSIS humanoid. Next, six joint angles (head, neck, torso, hip, knee, and ankle angles) of the humanoid were extracted from the reconstructed driving posture of RAMSIS humanoid (see Figure 2).

\subsection{Classification of sitting strategies by cluster analysis}

The sitting strategies based on the driving postures were identified in three steps (selection of proper number of clusters, classification of sitting strategies, and identification of sitting strategies). In the selection of proper number of cluster step, the number of clusters was selected based on a visual analysis of Euclidean distances between each cluster on a dendrogram plot from Ward's method. For example, as shown in Figure 3, the number of clusters $(n=3)$ for upper-body sitting strategies was selected based on the visual analysis of Euclidean distances which most likely distinguish between each cluster for upper-body joint angles. In the classification of sitting strategy step, the extracted driving postures were statistically classified by K-means cluster analysis using the selected number of clusters. Lastly, in the identification of sitting strategy step, the classified sitting strategies were identified to represent the characteristics of driving postures. 


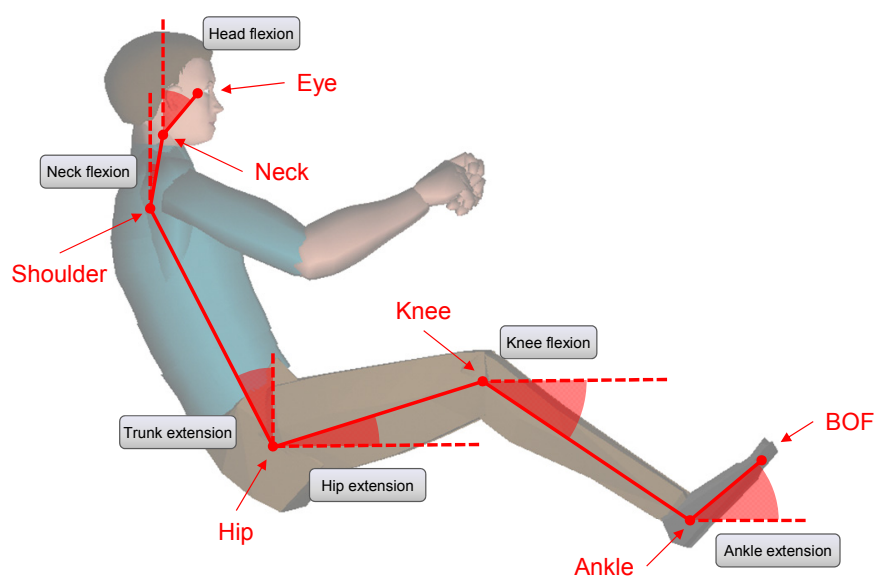

Figure 2. Selected joint angles for driving posture analysis

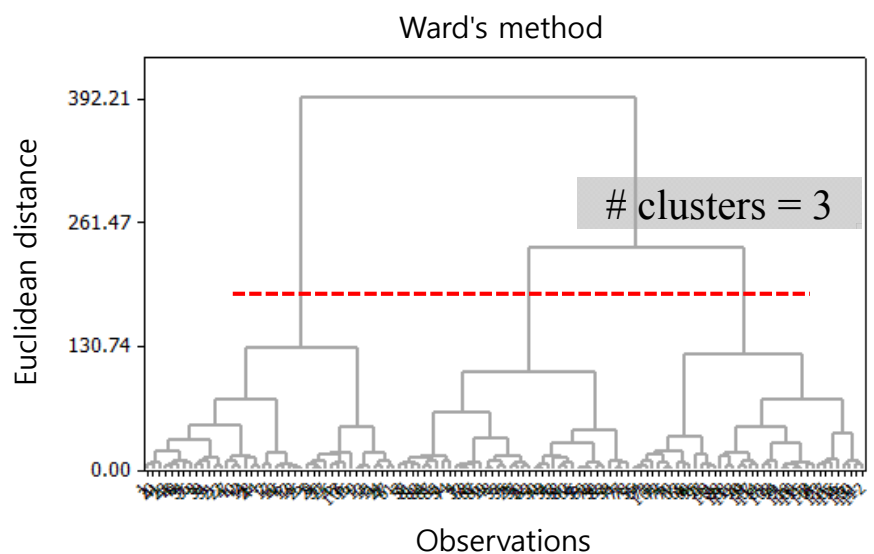

Figure 3. Selection of number of clusters using a dendrogram plot

\section{Results}

\subsection{Classification of sitting strategies}

The sitting strategies for upper-body (see Figure 4) were identified as slouched, erect, and reclined postures. The percentage of each upper-body sitting strategy was $41 \%$ for slouched, $33 \%$ for erect, and $26 \%$ for reclined posture.

The sitting strategies for lower-body (see Figure 5) were identified as knee bent, knee extended, and upper-leg lifted postures. The percentage of lower-body sitting strategies was $42 \%$ for knee bent, $32 \%$ for knee extended, and $26 \%$ for upper-leg lifted posture.

\subsection{Identification of related factors to the sitting strategies}

Driver's gender has significant effect on the upper-body sitting strategies $\left(\chi^{2}(2)=8.0, p<.05\right.$; see Figure $\left.6 \& 7\right)$. For example, $42.4 \%$ of female drivers preferred erect posture more than males (24.1\%); on the other hand, $36.2 \%$ of male drivers preferred 


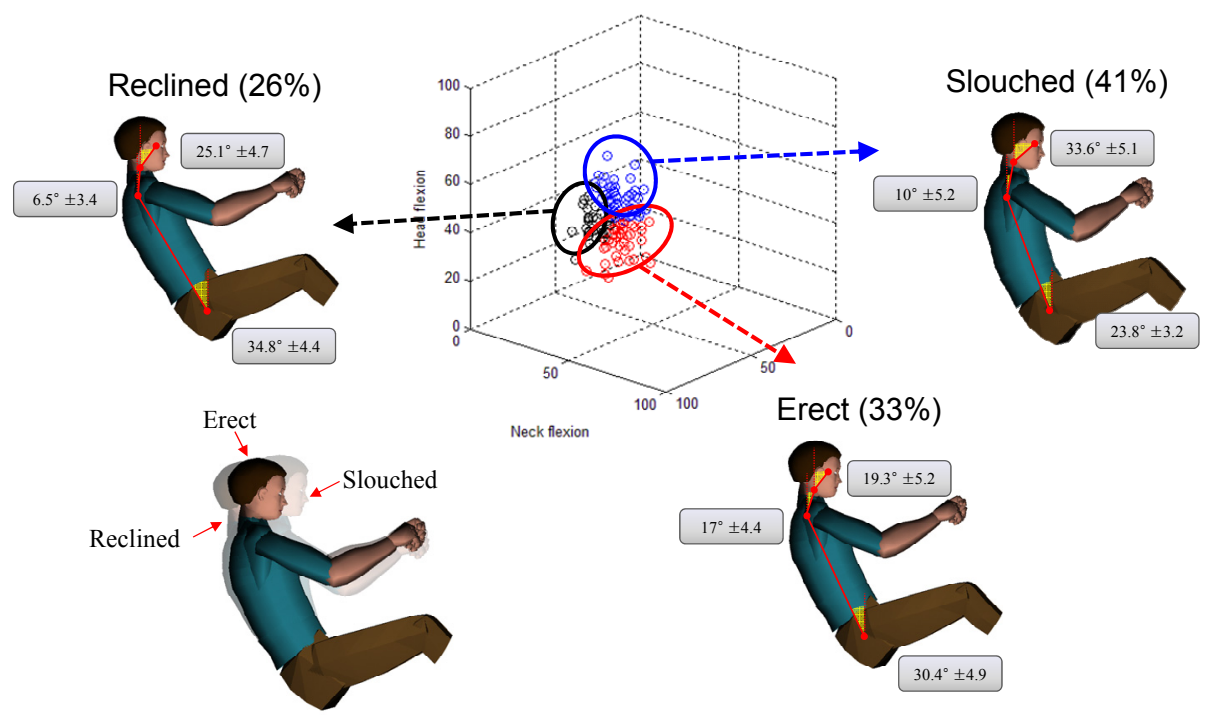

Figure 4. Classification of sitting strategy based on the upper-body driving posture

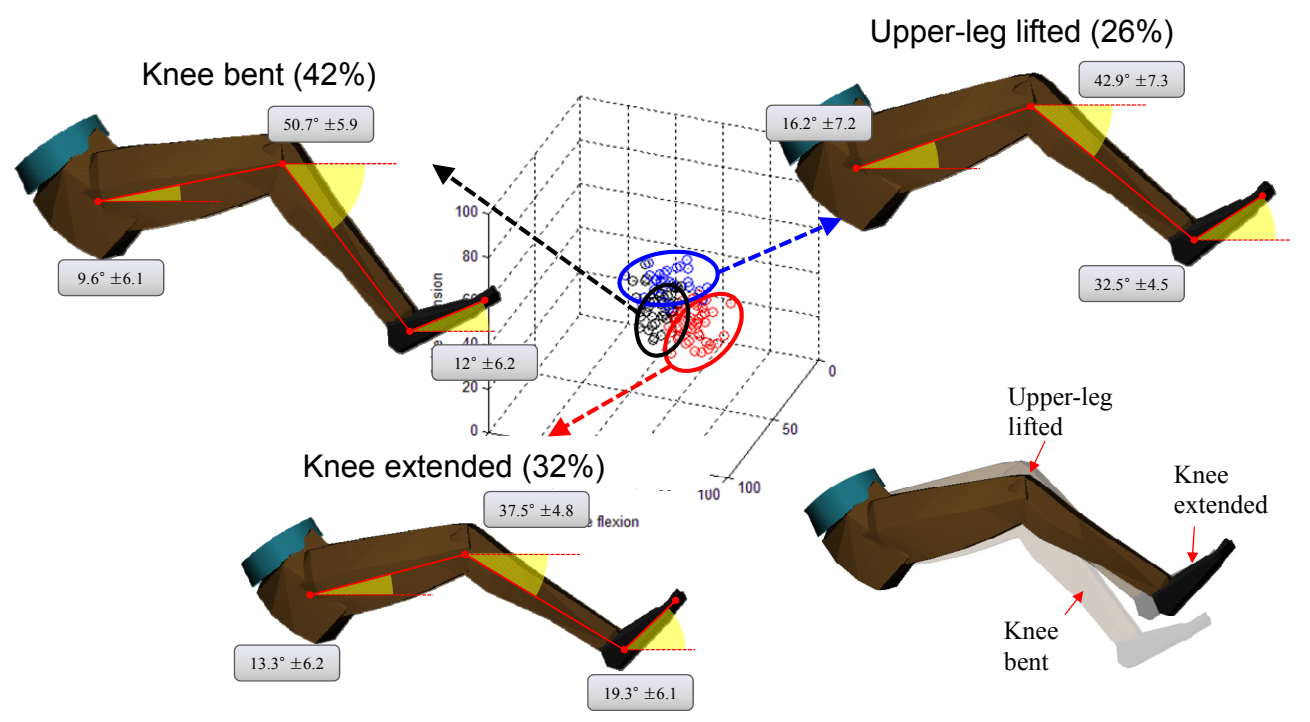

Figure 5. Classification of sitting strategies based on the lower-body driving posture

reclined posture more than females (15.3\%). There is no significant effect on lower-body sitting strategy by gender.

OPL condition has significant effect on the lower-body sitting strategies $\left(\chi^{2}(4)=56.3, p<.05\right.$, see Figure $\left.8 \& 9\right)$. For example, knee bent posture had hardly appeared at a coupe condition $(2.6 \%)$, nevertheless most of the participants took knee bent posture at the SUV condition (84.2\%). No significant effect was found on upper-body sitting strategy by OPL condition on the upper- \& lower-body sitting strategies by driver's stature (all $p \mathrm{~s}>.05$ ). 


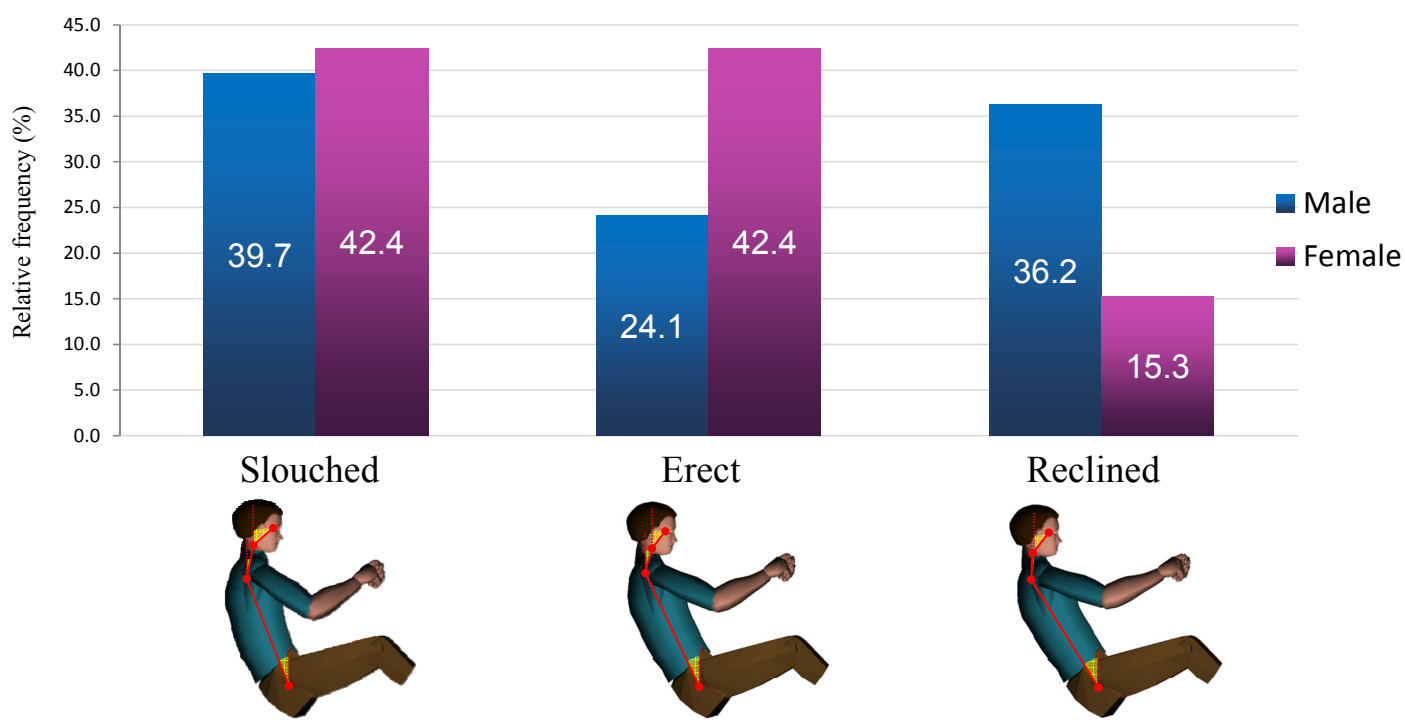

Figure 6. Percent of upper-body sitting strategies in gender $\left(\chi^{2}(2)=8.0, p=.019\right)$
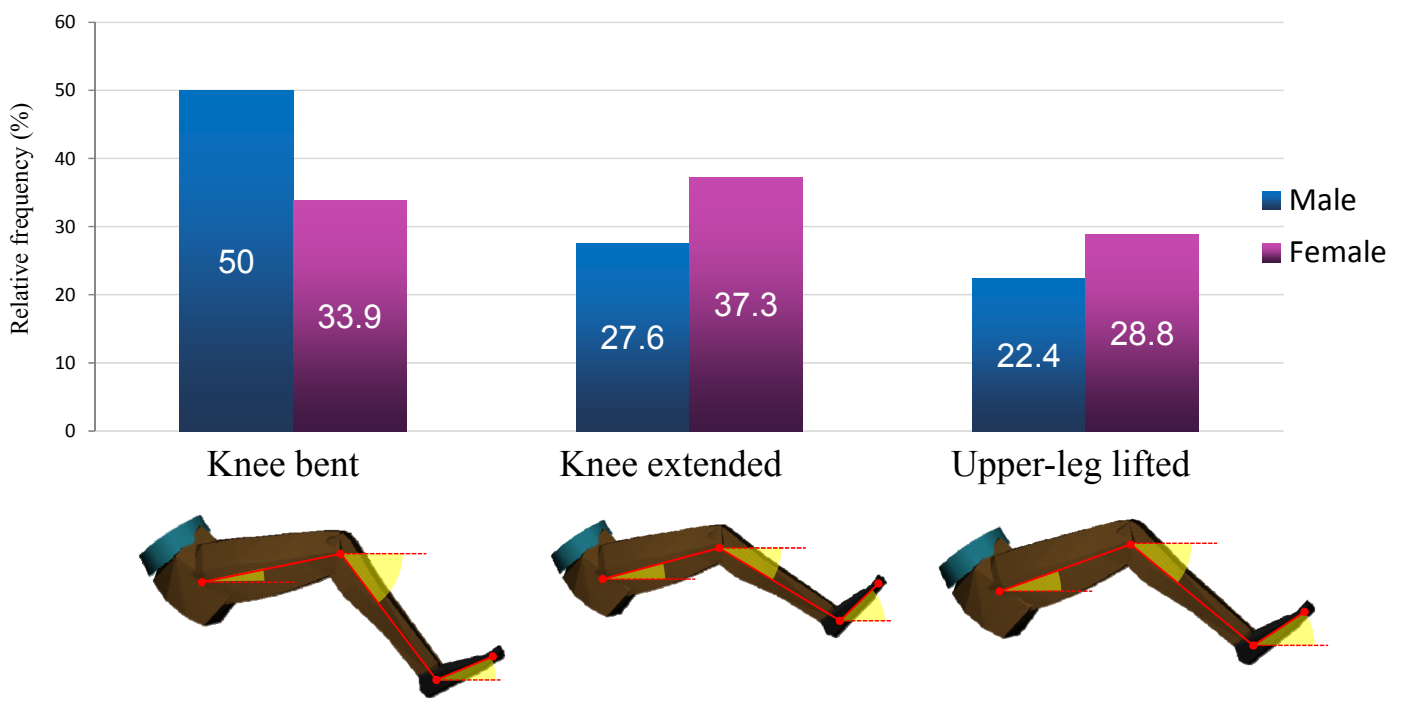

Figure 7. Percent of lower-body sitting strategies in gender $\left(\chi^{2}(2)=3.1, p=.21\right)$

\section{Discussion}

The present study statistically identified sitting strategies based on driving posture in order to design a driver workspace. The sitting strategy is particularly useful for vehicle interior design/evaluation using a digital human model in a virtual environment. However, Andreoni et al. (2002)'s sitting strategies is not objective due to visual classification. Although Park (2006)' sitting strategies were classified quantitatively and systematically using cluster analysis on joint angles, the factors (e.g., gender, OPL condition) for the sitting strategy were not clearly identified. On the other hand, this study objectively classified sitting strategies using cluster analysis and statistically identified factors for the sitting strategies. Therefore, the classified sitting strategies can be 


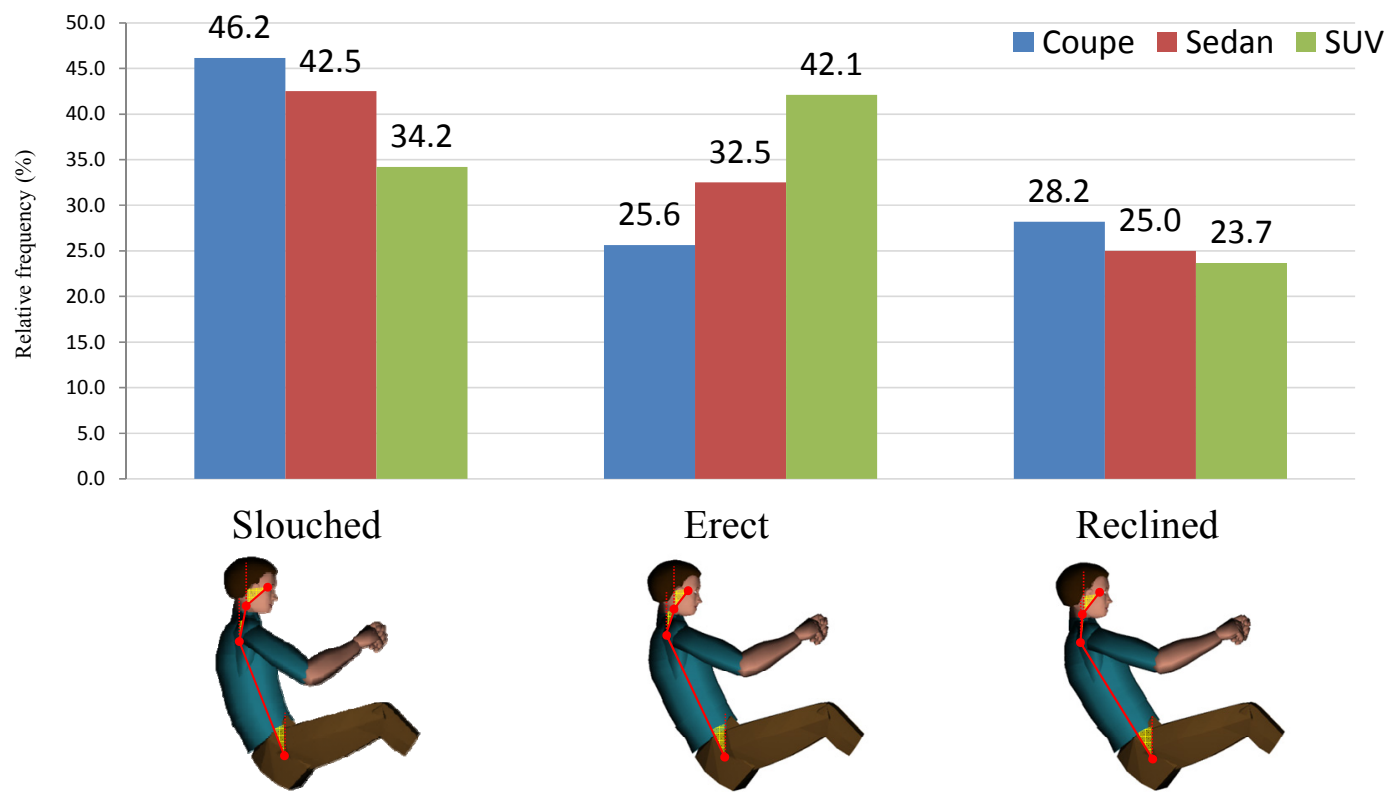

Figure 8. Percent of lower-body sitting strategies in OPL types $\left(\chi^{2}(4)=2.4, p=.66\right)$
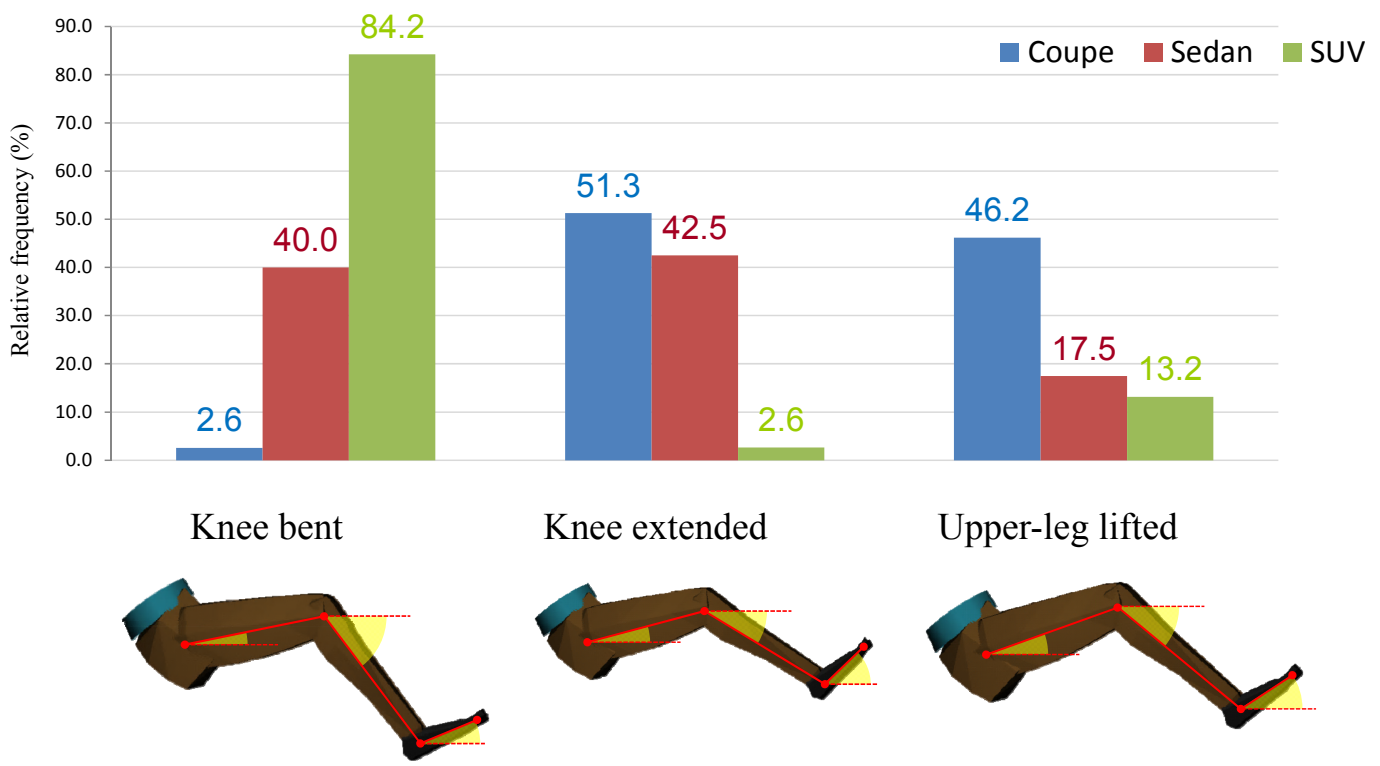

Figure 9. Percent of lower-body sitting strateies in OPL types $\left(\chi^{2}(4)=56.3, p<.01\right)$

used as a reference data for driver workspace design/evaluation using digital human model simulation at specific gender and OPL conditions.

The driver's gender has significant effect on the upper-body sitting strategies. This study revealed male drivers preferred posture strategies evenly (percentage of slouched posture $=39.7 \%$, erect posture $=24.1 \%$, and reclined posture $=36.2 \%$ ). However, 
female drivers prefer slouched or erect posture (percentage of slouched posture $=42.4 \%$, erect posture $=42.4 \%$, and reclined posture $=15.3 \%$ ). This result concludes that since arm-length of female drivers is relatively shorter than that of male drivers, female drivers moved their upper-body toward to the steering wheel to grasp steering wheel appropriately. Futhermore, since the sitting height of female drivers is relatively shorter than that of male drivers, female drivers might have moved their upperbody toward to the steering wheel to secure enough view. This difference of preferred driving postures depending on the driver's gender could be used for the selection of representative driving postures of female and male digital human models. For example, by selecting slouched posture and erect posture as representative postures for a female digital human model, the ergonomic evaluation of an automobile interior can be effectively performed.

The OPL condition has significant effect on lower-body sitting strategies. In this study, $84 \%$ of the participants preferred knee bent posture in the SUV condition; on the other hand, only $4 \%$ of the participants preferred knee bent posture in the coupe condition. This result implies that driver's lower-body posture is affected by seat height (H30). In the case of SUV condition (seat height $=305 \mathrm{~mm}$ ), drivers sit forward closer by pulling the seat location to the pedal and bend their knees to control the pedal comfortably. However, in the case of coupe condition (seat height $=176 \mathrm{~mm}$ ), it is hard to secure enough seat height to bend the knees; therefore, drivers might have moved seat location to backward and they preferred knee extended posture. This result also can be used in virtual automobile interior design process; for example, knee bent posture can be selected as a representative posture for lower-body of humanoid in design and evaluation of SUV.

\section{Acknowledgements}

This research was supported by the Basic Science Research Program through the National Research Foundation of Korea (NRF) funded by the Ministry of Education, Science, and Technology 2012-011373 and the Next Generation Vehicle (NGV).

\section{References}

Andreoni, G., Santambrogio, G.C., Rabuffetti, M. and Pedotti, A., Method for the analysis of posture and interface pressure of car drivers, Applied Ergonomics, 33, 511-522, 2002.

Cappozzo, A., Catani, F., Leardini, A., Benedetti, M.G. and Della Croce, U., Position and orientation in space of bones during movement: Experimental artifacts, Clinical Biomechanics, 11(2), 90-100, 1996.

Choi, Y., Park, J., Lee, B., Jung, K., Sah, S. and You, H., A classification of sitting strategies based on seating pressure distribution, Journal of the Korean Institute of Industrial Engineers, 39(2), 105-108, 2013.

Grieco, A., Sitting posture: an old problem and a new one, Ergonomics, 29(3), 345-362, 1986.

Kyung, G. and Nussbaum, M.A., Specifying comfortable driving postures for erfonomic design and evaluation of the driver workspace using digital human models, Ergonomics, 52(8), 939-953, 2009.

Park, J., Jung, K., Chang, J., Kwon, J. and You, H., Evaluation of predicted driving postures in RAMSIS digital human model simulation, IE Interfaces, 23(2), 101-108, 2010.

Park, S., Kim, C., Kim, C. and Lee, J., Comfortable driving postures for Koreans, International Journal of Industrial Ergonomics, 26, 489-497, 2000. 
Park, S., Estimation of driver's standard postures by a multivariate analysis method, Journal of the Ergonomics Society of Korea, 25(1), 27-33, 2006.

Reed, M.P., Manary, M.A., Flannagan, C.A.C. and Schneider, L.W., A statistical method for predicting automobile driving posture, Human Factors, 44(4), 557-568, 2002.

Ryu, T., A Direct Estimation of Anatomical Landmark Positions from Skin Markers by Identifying the Displacement Relationship (Unpublished doctoral dissertation), POSTECH, Pohang, South Korea, 2006.

Size Korea, Report on the Fifth Survey of Korean Anthropometry, Retrieved June 26, 2010 from http://sizekorea.kats.go.kr/

Speyer, H., RAMSIS Definition of Anthropometric Measurements, Human Solutions GmbH, Germany, 2005.

\section{Author listings}

Jangwoon Park: parkjw@postech.ac.kr

Highest degree: Ph.D., Department of Industrial and Management Engineering, POSTECH

Position title: Post-doctoral research associate, Department of Industrial \& Management Engineering, POSTECH

Areas of interest: Ergonomic product design \& development, User interface design \& evaluation, Digital human modeling \& simulation, Usability testing

Younggeun Choi: sidek@postech.ac.kr

Highest degree: M.S., Department of Industrial \& Management Engineering, POSTECH

Position title: Ph.D. candidate, Department of Industrial \& Management Engineering, POSTECH

Areas of interest: Digital human modeling \& simulation, User-centered product design \& development, Anthropometric and biomechanical methods for product development, Universal design, User experience design

Baekhee Lee: x200won@postech.ac.kr

Highest degree: M.S., Department of Industrial \& Management Engineering, POSTECH

Position title: Ph.D. candidate, Department of Industrial \& Management Engineering, POSTECH

Areas of interest: Ergonomic product design \& development, User interface, Usability testing, Sizing system, Vehicle ergonomics, Clinical ergonomics, Healthcare

Kihyo Jung: kjung@ulsan.ac.kr

Highest degree: Ph.D., Department of Industrial and Management Engineering, POSTECH

Position title: Assistant Professor, School of Industrial Engineering, University of Ulsan

Areas of interest: Ergonomic product design, Digital human simulation, Usability testing, Work-related musculoskeletal disorders (WMSDs) Prevention

Sungjin Sah: Sungjin.Sah@hyundai.com

Highest degree: Ph.D., Mechanical Engineering, Hongik University

Position title: Senior research engineer, Advanced Trim Engineering Team, Research \& Development Division, Hyundai Motor Company \& Kia Motors Corporation 
Areas of interest: Digital human body modeling, Human posture \& motion analysis

Heecheon You: hcyou@postech.ac.kr

Highest degree: Ph.D., Industrial Engineering, Pennsylvania State University

Position title: Professor, Department of Industrial and Management Engineering, POSTECH

Areas of interest: Ergonomic product design \& development, User interface design \& evaluation, Digital human modeling \& simulation, Human performance \& workload assessment, Work-related musculoskeletal disorders (WMSDs) prevention, Usability testing 